Pacific Journal of Mathematics

A BONNESEN-STYLE INRADIUS INEQUALITY IN 3-SPACE 


\section{A BONNESEN-STYLE INRADIUS INEQUALITY IN 3-SPACE}

\section{J. R. SANGWINE-YAGER}

A Bonnesen-style inradius inequality for convex bodies in $E^{3}$ is obtained using the method of inner parallel bodies. The inequality involves the volume, surface area and mean-width of the body.

I. Introduction. By a convex body we mean a compact convex set with non-empty interior. Let $K$ be a planar convex body with area $A$, perimeter $L$, inradius $r$, and circumradius $R$. An inequality of Bonnesen states:

$$
L^{2}-4 \pi A \geq \pi^{2}(R-r)^{2}
$$

This inequality follows from

$$
0 \geq A-x L+x^{2} \pi, \quad r \leq x \leq R .
$$

Equality holds in (1), at $x=r$, for the "sausage" bodies, that is, those bodies which are the Minkowski sum of a line segment and a ball (with radius $r$ ). At $x=R$ equality only holds for balls. For proofs of these inequalities see Eggleston [5, pp. 108-110].

An extension of Bonnesen's inradius inequality in the plane to higher dimensions began with the conjecture by Wills [11] that

$$
0 \geq V-r S+(n-1) r^{n} \omega_{n} .
$$

In this paragraph, $V$ will represent the $n$-dimensional volume of a convex body in $E^{n}, S$ its $n$-dimensional surface area, and $\omega_{n}$ the volume of the unit $n$-ball. The conjecture was proved simultaneously by Bokowski [1] and Diskant [4]. Equality holds only for the $n$-balls. Osserman [8] showed that

$$
0 \geq V-r S+(n-1) r^{2} \sqrt[n-1]{\omega_{n}(S / n)^{n-2}}
$$

where equality also holds only for the $n$-balls. This inequality is the sharper because a translate of $r B$ is contained in $K$.

The results of this paper will be limited to the case $n=3$. The volume and surface area of the convex body $K$ will be represented by $V(K)$ and $S(K)$. The unit 3-ball centered at the origin is denoted $B$ and $V(B)=\omega$. The functional $M(K)$ will be proportional to the 
mean-width of $K$, where $M(B)=3 \omega$. Often we will let $V=V(K)$, $S=S(K)$ and $M=M(K)$.

The following inequality for 3-dimensional bodies will be established:

$$
0 \geq V-r S+\frac{2}{3} r^{2} M
$$

where equality holds if and only if $K$ is a cap body of a ball. A cap body of a ball is the convex hull of the ball and countably many points exterior to it such that the line segment joining any two of these points intersects the ball. The cap bodies are also the extremal bodies for the inequality of Minkowski, $M^{2} \geq 3 S \omega$. From this inequality it follows that (3) is sharper than (2).

The inequality (3) is a consequence of a more general inequality for inner parallel bodies to be proved below. In proving our result we will actually establish a lower bound for the volume of the inner parallel bodies of a given body. This bound will resolve a conjecture of Matheron [7].

II. Main results. The inner parallel body of a convex body $K$ at a distance $\lambda \geq 0$ is defined to be the intersection of the closed supporting half-spaces of $K$ moved in a distance $\lambda$. The inradius $r$ is the radius of the largest ball contained in $K$. The inner parallel body at a distance $r-\lambda, 0 \leq \lambda \leq r$, shall be denoted $K_{\lambda}$; the inradius of $K_{\lambda}$ is $\lambda$. The kernel, $K_{0}$, is the locus of centers of balls of radius $r$ contained in $K$, and $K=K_{r}$.

The extremal bodies in the inequalities which follow are cap bodies. For the definition of $p$-extreme directions, $p=0,1$ or 2 , see Schneider $[9$, p. 135]. A supporting hyperplane is $p$-extreme if its outer normal is a $p$-extreme direction. A 0 -extreme direction is simply called extreme [5, p. 27]; the dual of the Krein-Milman Theorem states that every convex body is the intersection of its extreme supporting half-spaces. A convex body $K$ is a $(2-p)$-tangential body of the body $C$ if every $p$ extreme supporting hyperplane of $K$ also supports $C$. A 1-tangential body is a cap body; an alternate definition of a cap body of a ball is given above. A theorem of Favard [6, pp. 273-274] states that $K$ is a cap body of $C$ if and only if the mixed volumes $V(K, K, K)$, $V(K, K, C)$ and $V(K, C, C)$ are equal. The definition and properties of mixed volumes in general may be found in [5, p. 84]. In particular, $V(K)=V(K, K, K), S(K)=3 V(K, K, B), M(K)=3 V(K, B, B)$, and the mixed volumes are linear in each argument. 
We shall now prove the following

THEOREM. Let $K$ be a convex body in $E^{3}$ with non-empty interior and inradius $r$. For all $\lambda, 0 \leq \lambda \leq r$,

$$
V\left(K_{\lambda}\right) \geq V-(r-\lambda) S+(r-\lambda)^{2}\left[2 M+M\left(K_{\lambda}\right)\right] / 3
$$

where equality holds for all $\lambda, 0 \leq \lambda \leq r$, if and only if $K$ is a cap body of $K_{0}+r B$.

Proof of the Theorem. Fix $\lambda, 0<\lambda<r$. For all $t, \lambda \leq t \leq r$, let

$$
f(t)=V\left(K_{\lambda}\right)-V\left(K_{t}\right)+(t-\lambda) S\left(K_{t}\right)-(t-\lambda)^{2}\left[2 M\left(K_{t}\right)+M\left(K_{\lambda}\right)\right] / 3 .
$$

Bol [3] proved many results about functions of inner parallel bodies and their right- and left-hand derivatives. For example, $V\left(K_{t}\right)$ and $S\left(K_{t}\right)$ are absolutely continuous. If $F^{\prime}(t)$ denotes the left-hand derivative of any function $F(t)$ with respect to $t$, then

$$
\begin{aligned}
& V^{\prime}\left(K_{t}\right)=S\left(K_{t}\right), \\
& S^{\prime}\left(K_{t}\right) \geq 2 M\left(K_{t}\right) .
\end{aligned}
$$

The function $M\left(K_{t}\right)$ is a concave function of $t$ which implies

$$
M\left(K_{t}\right)-M\left(K_{\lambda}\right) \geq(t-\lambda) M^{\prime}\left(K_{t}\right) .
$$

Using (5) we find

$f^{\prime}(t)=(t-\lambda)\left[\left[S^{\prime}\left(K_{t}\right)-2 M\left(K_{t}\right)\right]+2\left[M\left(K_{t}\right)-M\left(K_{\lambda}\right)-(t-\lambda) M^{\prime}\left(K_{t}\right)\right] / 3\right]$.

Therefore by (6) and (7) $f^{\prime}(t) \geq 0 ; f(\lambda)=0$, implies $f(r) \geq 0$. This establishes the inequality.

It remains to establish the equality condition. Favard's Theorem states that $K$ is a cap body of $K_{0}+r B$ if and only if

$$
V\left(K, K_{0}+r B, K_{0}+r B\right)=V\left(K, K, K_{0}+r B\right) .
$$

If equality holds in (4) for all $\lambda, 0 \leq \lambda \leq r$, then for $\lambda$ fixed, $0<$ $\lambda<r, f(t)$ must be identically zero. Therefore equality must hold in (6) and (7) for all $\lambda$ and $t, 0<\lambda<t \leq r$. It should be noted that the conditions for equality in (6) remain unknown since they were first investigated by Bol [3]. Equality in (7) implies that $M\left(K_{t}\right)$ is a linear function of $t$. Since families of parallel bodies are concave, we have

$$
\frac{t}{r} K+\left(1-\frac{t}{r}\right) K_{0}=K_{t},
$$

for all $t, 0 \leq t \leq r$. The representation for $K_{t}$ in (9) may be used to 
expand and differentiate $S\left(K_{t}\right)$ :

$$
\begin{gathered}
S\left(K_{t}\right)=\left(\frac{t}{r}\right)^{2} S(K)+6 \frac{t}{r}\left(1-\frac{t}{r}\right) V\left(K, K_{0}, B\right)+\left(1-\frac{t}{r}\right)^{2} S\left(K_{0}\right), \\
\text { (10) } \frac{r}{2} S^{\prime}\left(K_{t}\right)=\frac{t}{r} S(K)+3\left(1-\frac{2 t}{r}\right) V\left(K, K_{0}, B\right)-\left(1-\frac{t}{r}\right) S\left(K_{0}\right) .
\end{gathered}
$$

At $t=r$,

$$
\frac{r}{2} S^{\prime}(K)=S(K)-3 V\left(K, K_{0}, B\right) .
$$

Substitution into (6), with equality, gives

$$
S(K)-3 V\left(K, K_{0}, B\right)=r M(K)
$$

or

$$
V(K, K, B)=V\left(K, K_{0}+r B, B\right) \text {. }
$$

The expression in (11) implies that the supporting functions of $K$ and $K_{0}+r B$ are equal on the support of the mixed area measure $S(K, B ; \cdot)$. Results of Schneider [9, pp. 134-135] imply (8).

On the other hand, suppose $K$ is a cap body of $K_{0}+r B$. Since the inner parallel bodies of $K$ are the intersection of the extreme supporting half-spaces of $K$ moved inward, the extreme directions of an inner parallel body are extreme directions of $K$. Choose $\lambda$ and $t$, $0<\lambda<t<r$, let $\theta=(t-\lambda) /(r-\lambda)$, and let $u$ be a unit vector. If $h(C, u)$ is the supporting function of a convex body $C$ in the direction $u$, then

$$
\text { (12) } K_{0}+t B \subseteq \theta K+(1-\theta) K_{\lambda} \subseteq K_{t} \subseteq\{x:\langle x, u\rangle \leq h(K, u)-(r-t)\} \text {. }
$$

An extreme direction of $\theta K+(1-\theta) K_{\lambda}$ is either an extreme direction of $K$, an extreme direction of $K_{\lambda}$ (and hence $K$ ), or a 1extreme direction of both. If $u$ is a 0 - or 1-extreme direction of $K$, then $h(K, u)=h\left(K_{0}, u\right)+r$, and the supporting half-space in (12) also supports $K_{0}+t B$. Therefore equality holds throughout (12), and the representation in (9) is valid. This implies equality in (7). The result of Favard (8) has many implications. It implies both (11) and $V\left(K, K, K_{0}\right)=V\left(K, K_{0}+r B, K_{0}\right)$. Furthermore, since the support of the mixed area measure $S\left(B, K_{\lambda} ; \cdot\right)$ is the closure of the set of extreme directions of $K_{\lambda}, V\left(K, B, K_{\lambda}\right)=V\left(K_{0}+r B, B, K_{\lambda}\right)$. Hence $V\left(K, B, K_{0}\right)=V\left(K_{0}+r B, B, K_{0}\right)$. Substitution into the right-hand side of (10) yields

$$
\frac{r}{2} S^{\prime}\left(K_{t}\right)=3\left(t V(K, B, B)+(r-t) V\left(K_{0}, B, B\right)\right) .
$$

Equality holds in (6) also. This completes the proof of the theorem. 
For $\lambda=0$, (4) becomes the following Bonnesen-style inequality

$$
0 \geq V-r S+r^{2}\left[2 M+M\left(K_{0}\right)\right] / 3
$$

which implies (3). Equality holds if and only if $K$ is a cap body of $K_{0}+r B$, and therefore in (3) if and only if $K$ is a cap body of a ball. The cap bodies appear in the equality condition for (3) because they are precisely those bodies for which $3 V=r S=r^{2} M$.

Matheron [7] showed that in the plane

$$
A\left(K_{\lambda}\right) \geq A-(r-\lambda) L+(r-\lambda)^{2} \pi
$$

where equality holds for all $\lambda, 0 \leq \lambda \leq r$, if and only if $K$ is a sausage body. Notice that at $\lambda=0$ this inequality is (1), for $x=r$, since the area of the kernel is zero.

The right-hand side of Matheron's inequality is similar to Steiner's formula for the outer parallel bodies of $K$, except that the signs alternate. Matheron conjectured that similar lower bounds existed for the volume of the inner parallel bodies in higher dimensional spaces. Weil [10] has established necessary and sufficient conditions for one body to be the summand of another. This result implies that if $K_{\lambda}+(r-\lambda) B=K$, for $K$ in $E^{3}$, then

$$
V\left(K_{\lambda}\right)-\left[V-(r-\lambda) S+(r-\lambda)^{2} M-(r-\lambda)^{3} \omega\right]=0 .
$$

The conjectured inequality does not exist because the difference above is positive, at $\lambda=r$, for the cubes and negative for cap bodies. Notice that the right-hand side of (4) is equal to

$$
V-(r-\lambda) S+(r-\lambda)^{2} M-(r-\lambda)^{3} \omega+(r-\lambda)^{2}\left[M\left(K_{\lambda}+(r-\lambda) B\right)-M\right] / 3
$$

which is smaller than the alternating sum proposed by Matheron because $K_{\lambda}+(r-\lambda) B \subseteq K$.

III. Further remarks. Remarks will be made on two significant differences between (3) and (1): inequality (3) does not strengthen the isoperimetric inequality, and it is not valid if the inradius $r$ is replaced with the circumradius $R$.

For $n>2$, sharpened versions of the isoperimetric inequality have been established, and Osserman [8, pp. 24-25] shows that these inequalities imply (2). Inequality (3) does not yield either isoperimetricstyle inequality

For example, (3) implies

$$
S^{2} \geq 3 V M \text { or } S^{3} \geq 27 V \omega .
$$

$$
S^{2}-3 V M \geq\left(\frac{V}{r}\right)^{2}-\frac{5}{3} V M+\frac{4}{9} r^{2} M^{2},
$$


but the right-hand side is negative for pencil-like bodies. This raises the question of whether (3) is a strong inequality. It is if we view (1), for $x=r$, and (3) in the following sense: They provide the best possible lower bounds by weighted arithmetic means for $r L$ and $r S$ respectively. In (3) $r S$ is bounded below by $\theta 3 V+(1-\theta) r^{2} M$. This bound is best when $\theta$ is minimized ( $\theta=1$ for the cube). The value of $\theta=1 / 3$ is approached in the limit for a family of cylinders which converge to a disk in the plane.

Recently, Bonnesen-style circumradius inequalities involving three quermassintegrals in $n$-dimensional space have been found by Bokowski and Heil [2]. One inequality, for $n=3$, is

$$
0 \leq V-4 R S / 3+R^{2} M \text {. }
$$

Equality holds if and only if $K$ is homothetic to $R B$. Notice that the direction of the inequality is reversed from (1) and (3). The inequality is also a bound for $R S$ by a weighted arithmetic mean, and it has been shown to be best.

\section{REFERENCES}

[1] J. Bokowski, Eine verschärfte Ungleichung zwischen Volumen, Oberfläche und Inkugelradius im $R^{n}$, Elem. Math., 28 (1973), 43-44.

[2] J. Bokowski and E. Heil, Integral representations of quermassintegrals and Bonnesen-Style inequalities, Arch. Math., to appear.

[3] G. Bol, Beweis einer Vermutung von H. Minkowski, Abh. Math. Sem. Univ. Hamburg, 15 (1943), 37-56.

[4] V. I. Diskant, $A$ generalization of Bonnesen's inequalities, Soviet Math. Dokl., 14 no. 6 (1973), 1728-1731 (Transl. of Dokl. Akad. Nauk SSSR, 213 (1973), no. 3).

[5] H. G. Eggleston, Convexity, Cambridge U. Press, Cambridge, 1969.

[6] J. Favard, Sur les corps convexes, J. Math. Pur. Appl., IX. Sér., 12 (1933), 219-282.

[7] G. Matheron, La formule de Steiner pour les érosions, J. Appl. Prob., 15 (1978), 126-135.

[8] R. Osserman, Bonnesen-Style isoperimetric inequalities, Amer. Math. Monthly, 86, no. 1, (1979), 1-29.

[9] R. Schneider, On the Aleksandrov-Fenchel inequality, Discrete Geometry and Convexity, New York Academy of Sciences, (1985), 132-141.

[10] W. Weil, Decomposition of convex bodies, Mathematika, 21 (1974), 19-25.

[11] J. M. Wills, Zum Verhaltnis von Volumen zur Oberfläche bei konvexen Korpern, Arch. Math., 21 (1970), 557-560.

Received July 6, 1987. This work was supported in part by the Saint Mary's College Faculty Development Fund. 


\section{PACIFIC JOURNAL OF MATHEMATICS EDITORS}

V. S. VARADARAJAN (Managing Editor) University of California Los Angeles, CA 90024

HeRbert Clemens

University of Utah

Salt Lake City, UT 84112

R. FINN

Stanford University

Stanford, CA 94305
ROBION KIRBY

University of California

Berkeley, CA 94720

C. C. MOORE

University of California

Berkeley, CA 94720

HAROLD STARK

University of California, San Diego

La Jolla, CA 92093

\section{ASSOCIATE EDITORS}
R. ARENS
E. F. BECKENBACH
B. H. NEUMANN
F. Wolf
K. YOSHIDA (1906-1982)

\section{SUPPORTING INSTITUTIONS}

UNIVERSITY OF ARIZONA UNIVERSITY OF OREGON

UNIVERSITY OF BRITISH COLUMBIA UNIVERSITY OF SOUTHERN CALIFORNIA

CALIFORNIA INSTITUTE OF TECHNOLOGY UNIVERSITY OF CALIFORNIA MONTANA STATE UNIVERSITY STANFORD UNIVERSITY

UNIVERSITY OF NEVADA, RENO UNIVERSITY OF HAWAII NEW MEXICO STATE UNIVERSITY UNIVERSITY OF TOKYO UNIVERSITY OF UTAH OREGON STATE UNIVERSITY WASHINGTON STATE UNIVERSITY UNIVERSITY OF WASHINGTON 


\section{Pacific Journal of Mathematics}

Vol. 134, No. $1 \quad$ May, 1988

Marco Abate, Annular bundles . ............................

Ralph Cohen, Wen Hsiung Lin and Mark Mahowald, The Adams

spectral sequence of the real projective spaces

Harry Joseph D'Souza, Threefolds whose hyperplane sections are elliptic surfaces .........................................57

Theodore Gerard Faticoni, Localization in finite-dimensional FPF rings . . . 79

Daniel Hitt, Invariant subspaces of $\mathscr{H}^{2}$ of an annulus ................ 101

Ellen Kirkman and James J. Kuzmanovich, On the global dimension of fibre products

Angel Rafael Larotonda and Ignacio Zalduendo, Homogeneous spectral sets and local-global methods in Banach algebras

Halsey Lawrence Royden, Jr., Invariant subspaces of $\mathscr{H}^{p}$ for multiply

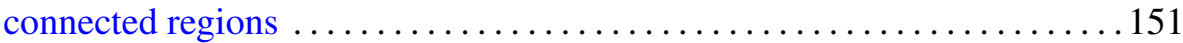

Jane Sangwine-Yager, A Bonnesen-style inradius inequality in 3-space . . . . 173

Stefano Trapani, Holomorphically convex compact sets and cohomology

Thomas Vogel, Uniqueness for certain surfaces of prescribed mean

curvature 